\title{
МОРФОФУНКЦИОНАЛЬНЫЕ ОСОБЕННОСТИ СТРОЕНИЯ СЕРДЦА У ДЕТЕЙ ГРУДНОГО ВОЗРАСТА ПО ДАННЫМ УЛЬТРАЗВУКОВОГО ИССЛЕДОВАНИЯ
}

\begin{abstract}
С каждым годом медицина принципиально меняется, и в основе этого лежит стремительное развитие аппаратной диагностики. Современные методы ультразвуковых исследований позволяют сравнивать функциональные показатели сердца в разные периоды постнатального онтогенеза, что является важным в прогнозировании заболеваемости при адекватном развитии целостного организма $[1,2]$.

Но, несмотря на множество работ, посвященных сердечно-сосудистой системе, и опубликованных эхокардиографических данных, они являются противоречивыми и не учитывают особенности конкретного региона [3-5].

Это требует разработки региональных эхокардиографических справок. Для интерпретации количественных эхокардиографических данных размеры сердца должны сравниваться с нормальными значениями. [6, 7]. Необходимо постоянное обновление количественных данных методом ультразвуковой диагностики с учетом этнической и региональной принадлежности детей.
\end{abstract}

\section{ЦЕЛЬРАБОТ Ы}

Установить количественные показатели объемных и гемодинамических параметров сердца и магистральных сосудов в области его основания у детей грудного возраста Донецкого региона.

\section{МАТЕРИАЛ И МЕТОДЫ}

Исследования проведены на 80 детях в возрасте от одного месяца до года, у которых отсутствовали признаки заболеваний сердечнососудистой системы и другой соматической патологии. Они были поделены на две группы по половому признаку. Первую группу составили 42 ребенка мужского пола, вторую группу - 38 женского. Методом ультразвукового исследования сердца в М- и В-режимах измеряли и оценивали конечный диастолический (КДО) и конечный систолический объемы (КСО) левого желудочка, фракцию выброса (ФВ). Допплеровским методом исследовали скорость потоков крови в устье аорты, легочного ствола, правого и левого предсердно-желудочкового устья и их градиент давления.

Все количественные параметры обрабатывали статистическими методами. Данные представлены в тексте в виде среднеарифметического значения и ошибки средней, медианы с минимальными и максимальными значениями, а также вносились в таблицы в виде $\mathrm{Me}(\mathrm{Q} 1-\mathrm{Q} 3)$, где Ме - медиана, Q1 - первый квартиль и Q3 третий квартиль. Полученные результаты проверены на нормальность (обе группы), закон распределения отличается от нормального на уровне значимости $\mathrm{p}<0,05$. Проведено сравнение центральных тенденций двух независимых выборок по критерию Манна-Уитни. В ходе исследования был проведен корреляционный анализ Спирмена.

\section{РЕЗУЛЬТАТЫ И ОБСУЖДЕНИЕ}

Анализ антропометрических показателей детей грудного возраста (см. табл.) показал, что средние значения веса тела и ошибка средней у

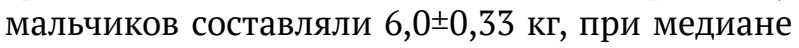
6,1 кг. Минимальные и максимальные показатели колебались в пределах 3,4-9,5 кг. Средние значения веса тела у девочек достигали $5,53 \pm 0,3$ кг с медианой 5,0 кг, при минимальных параметрах 3,2 кг и максимальных - 9,3 кг.

Средние значения роста у мальчиков выше, чем у девочек, $-65,45 \pm 1,26$ см, медиана составляла 65,0 см, при колебании роста от минимального - 49,0 см до максимального - 81,0 см. У девочек средние значения роста достигали $61,92 \pm 1,15$ см, а медиана - 60,0 см, при их минимальном росте 51,0 см и максимальном 85,0 см.

(c) В.А. Васильев, К.А. Мурейси, Р.В. Басий,

И.П. Вакуленко, И.В. Коктышев, 2020

(c) Университетская Клиника, 2020 
Объемные и гемодинамические показатели структур сердца

Таблица. в группах наблюдения по данным УЗИ $\mathrm{Me}(\mathrm{Q} 1-\mathrm{Q} 2)$

\begin{tabular}{lcc}
\hline \multirow{2}{*}{ Показатели } & \multicolumn{2}{c}{ Дети грудного возраста 1-12 мес., $\mathrm{n}=80$} \\
\cline { 2 - 3 } & $\begin{array}{c}\text { мальчики } \mathrm{n}=42 \\
\text { 1группа }\end{array}$ & $\begin{array}{c}\text { девочки } \mathrm{n}=38 \\
\text { 2группа }\end{array}$ \\
\hline \hline Вес, кг & $6,1(3,9-8,0)$ & $5,0(3,9-6,5)$ \\
\hline Рост, см & $65,0(60,0-71,0)$ & $60,0(58,0-65,0)$ \\
\hline Площадь тела, м & $0,28(0,23-0,37)$ & $0,27(0,23-0,33)$ \\
\hline КДО, мл & $14,8(12,8-17,7)$ & $14,6(12,2-16,9)$ \\
\hline КСО, мл & $3,85(3,56-6,0)$ & $3,86(3,39-4,55)$ \\
\hline ФВ \% & $68(64-72)$ & $67(63-72)$ \\
\hline Аортальный поток, см/сек & $108,8(98,8-114,4)$ & $104,6(99,1-117,0)$ \\
\hline Градиент давления РтmHg & $4,33(3,8-4,84)$ & $4,18(3,81-4,45)$ \\
\hline Митральный поток, см/сек & $95,8(84,4-103,2)$ & $90,35(81,5-98,9)$ \\
\hline Градиент давления РmmHg & $3,67(3,29-4,23)$ & $3,37(2,87-3,95)$ \\
\hline Легочный поток, см/сек & $97,25(92,2-100,0)$ & $96,6(89,99-100,5)$ \\
\hline Градиент давления РmmHg & $3,67(3,5-3,92)$ & $3,57(3,42-3,93)$ \\
\hline Трикуспидальный клапан, см/сек & $67,67(57,6-74,8)$ & $68,6(61,3-77,0)$ \\
\hline Градиент давления РmmHg & $1,83(1,32-2,24)$ & $1,76(1,38-2,34)$ \\
\hline
\end{tabular}

Площадь тела у лиц мужского пола достигала $0,30 \pm 0,08 \mathrm{~m}^{2}$, а $\mathrm{Me}=0,28 \mathrm{~m}^{2}$ при минимальных значениях $0,18 \mathrm{~m}^{2}$ и максимальных $-0,55 \mathrm{~m}^{2}$. У лиц женского пола средние значения площади тела составили $0,28 \pm 0,08 \mathrm{M}^{2}$, и медиана $-0,27 \mathrm{M}^{2}$ с колебанием параметров площади тела между 0,14-0,57 $\mathrm{M}^{2}$.

Были проанализированы объемные показатели детей грудного возраста, по данным ультразвукового исследования средние значения конечного диастолического объема левого желудочка у детей первой группы колебался в интервале 7,37- 30,2 мл. Его средние значения зафик-

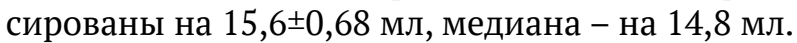
Во второй группе средние значения конечного диастолического объема составили $14,7 \pm 0,84$ мл, медиана при этом - 14,6 мл, при минимальном параметре 6,84 мл и максимальном - 38,8 мл.

Средние значения конечного систолического объема в первой группе - 4,62 $\pm 0,25$ мл, при медиане 3,85 мл и интервале между минимальным и максимальным параметрами от 2,35 до 8,86 мл. Во второй группе средние значения конеч-

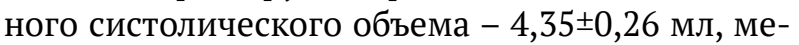
диана достигала 3,86 мл при минимальном значении 2,4 мл, а максимальном - 13,2 мл.

Фракция выброса у мальчиков достигала $68 \pm 0,8 \%$, и этот параметр совпадал с медианой. Интервал колебаний находился в пределах от
58 до 82\%. У девочек средние значения и медиана фракции выброса составили $67 \pm 0,1 \%$ с минимальными и максимальными параметрами от 53 до $83 \%$.

Средние значения скорости потока в устье аорты у девочек грудного возраста составляли $111,8 \pm 2,9$ cм/сек, медиана - 104,6 cм/сек, при минимальных значениях 91,9 см/сек и максимальных - 177,0 см/сек. У мальчиков средние значения скорости потока в устье аорты зафиксированы на 106,9 2,09 см/сек при медиане 108,8 cм/ сек. Минимальные параметры этого показателя соответствовали $71,3 \mathrm{~cm} / \mathrm{cek,} \mathrm{а} \mathrm{максимальные} \mathrm{-}$ $131,0 \mathrm{~cm} / \mathrm{ceк.}$

Градиент давления в устье аорты у лиц муж-

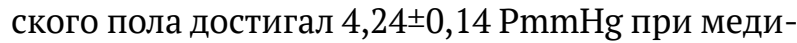
ане $4,33 \mathrm{PmmHg}$ с интервалом колебания давления между 2,03 и 5,8 PmmHg. Средние значения и медиана градиента давления у лиц женского пола совпадали на уровне $4,18 \pm 0,08 \mathrm{PmmHg}$. Минимальный параметр при этом составлял 3,25 $\mathrm{PmmHg}$, а максимальный достигал 5,44 PmmHg.

Скорость потока в левом предсердножелудочковом устье у мальчиков составляла 93,72 $\pm 2,2$ см/сек с медианой 95,8 cм/сек, интервал колебаний скорости потока фиксировали между 61,6 и 119,5с м/сек. У девочек средние значения скорости потока в левом предсердножелудочковом устье были меньше - 90,61士2,06 
см/сек, при медиане 90,35 cм/сек и минимальных значениях 68,4 см/сек, а максимальных 122,0 cм/сек.

Градиент давления в левом предсердно-желудочковом устье у мальчиков достигал 3,74ะ0,1 $\mathrm{PmmHg}$ с медианой 3,67 PmmHg. Минимальный показатель достигал 1,52 Pmm $\mathrm{Hg}$, а максимальный - 5,71 PmmHg. Средние значения градиента давления у девочек фиксировали на $3,45 \pm 0,1$ $\mathrm{PmmHg}$, при медиане 3,37 PmmHg и интервале колебаний между 1,87 и 5,93 PmmHg.

Средние значения скорости потока в легоч-

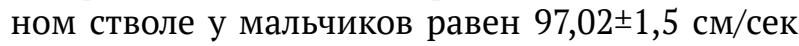
и совпадает с медианой 97,25 cм/сек. Вариабельность параметра находилась в пределах 75,4122,4 cм/сек. У девочек средние значения скорости потока в легочном стволе не отличались и приравнивались к 97,43ะ1,6 см/сек при медиане 96,6 cм/сек и интервале колебаний параметра от 83,0 до 123,0 см/сек.

Градиент давления в легочном стволе и у мальчиков, и у девочек отличался в незначительной степени. Средние значения в первой группе доходили до отметки 3,73 $\pm 0,1 \mathrm{PmmHg}$ с медианой 3,67 PmmHg при минимальном параметре 2,27 PmmHg и максимальном 5,56 Pm$\mathrm{mHg}$. Во второй группе градиент давления колебался в пределах от 2,88 до 5,09 PmmHg со средним значением $3,7 \pm 0,07 \mathrm{PmmHg}$ и медианой 3,57 PmmHg.

Скорость потока крови в правом предсердножелудочковом отверстии у лиц мужского пола

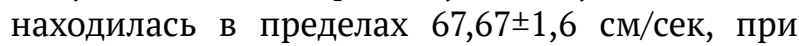
этом медиана не отличалась и соответствовала 67,67 cм/сек. Минимальные значения скорости потока в правом предсердно-желудочковом отверстии составляли $43,3 \mathrm{~cm} / \mathrm{ceк,} \mathrm{а} \mathrm{максимальные}$ достигали 87,6 cм/сек. У лиц женского пола скорость потока варьировала в пределах от 44,3 до 89,2 cм/сек. Средние значения были отмечены

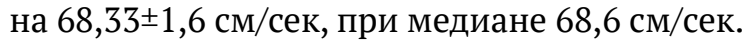

Градиент давления правого предсердно-желудочкового отверстия у мальчиков варьировал от 1,02 до 2,95 PmmHg. Средние значения находились на отметке $1,89 \pm 0,08 \mathrm{PmmHg}$, а медиана - на 1,83 PmmHg. У девочек средние значения

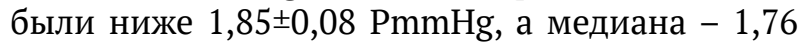
$\mathrm{PmmHg}$, при этом колебания градиента давления находились в пределах 1,05-2,75 PmmHg.

В ходе исследования выявлена положительная корреляционная связь средней силы в группе мальчиков грудного возраста между антропометрическими данными и объемными параметрами: весом и конечным диастолическим объемом $(\mathrm{R}=0,337 ; \mathrm{p}=0,04)$, ростом и конечным диастолическим объемом ( $\mathrm{R}=0,330 ; \mathrm{p}=0,03)$, возрастом и конечным диастолическим объемом $(\mathrm{R}=$
0,369; p=0,01), площадью тела и конечным диастолическим объемом $(\mathrm{R}=0,312 ; \mathrm{p}=0,04)$, весом и конечным систолическим объемом ( $\mathrm{R}=0,455$; $\mathrm{p}=0,002)$, ростом и конечным систолическим объемом (R=0,508; $\mathrm{p}=0,0005)$, возрастом и конечным систолическим объемом $(\mathrm{R}=0,457 ; \mathrm{p}=0,002)$, площадью тела и конечным систолическим объемом $(R=0,324 ; p=0,04)$.

Между скоростными показателями и антропометрическими данными корреляционной связи у мальчиков грудного возраста не выявлено.

У девочек этой же подгруппы также выявлена положительная корреляционная связь средней силы между антропометрическими данными и объемными параметрами: весом и конечным диастолическим объемом $(\mathrm{R}=0,352 ; \mathrm{p}=0,03)$, ростом и конечным диастолическим объемом $(\mathrm{R}=0,431 ; \mathrm{p}=0,03)$, возрастом и конечным диастолическим объемом $(\mathrm{R}=0,345 ; \mathrm{p}=0,03)$, площадью тела и конечным диастолическим объемом $(\mathrm{R}=0,410 ; \mathrm{p}=0,009)$, весом и конечным систолическим объемом $(\mathrm{R}=0,555 ; \mathrm{p}=0,0002)$, ростом и конечным систолическим объемом ( $\mathrm{R}=0,449$; $\mathrm{p}=0,004)$, возрастом и конечным систолическим объемом (R=0,583; $\mathrm{p}=0,0001)$, площадью тела и конечным систолическим объемом ( $\mathrm{R}=0,534$; $\mathrm{p}=0,0005)$.

Выявлена положительная корреляционная связь слабой силы между ростом и скоростью кровотока в левом предсердно-желудочковом устье $(\mathrm{R}=0,353 ; \mathrm{p}=0,02)$. Через остальные устья связи между показателями сердца с весом, ростом, площадью тела, возрастом детей не выявлено.

В ходе работы положительная корреляционная связь средней силы наблюдалась в обеих группах детей грудного возраста между весом, ростом, возрастом и площадью тела детей с конечным диастолическим и систолическим объемами левого желудочка. Обращает на себя внимание отсутствие корреляционной связи: в обеих группах по фракции выброса и у скоростных показателей корреляционная связь средней силы выявлена только у девочек между ростом и скоростью в левом предсердно-желудочковом устье.

Проведено сравнение центральных тенденций двух независимых выборок для всех показателей: вес, рост, площадь тела детей, конечный диастолический и систолический объемы левого желудочка, фракция выброса, скорость потока в устье аорты и легочного ствола, а также в правом и левом предсердно-желудочковом устье по критерию Манна-Уитни. Выявленные отличия не являются статистически значимыми на уровне значимости $\mathrm{p}>0,05$. 


\section{В Ы В О Д Ы}

Таким образом, в грудном возрасте при УЗИ структур сердца объемные показатели сердца интенсивнее нарастают у лиц женского пола, в то время как гемодинамические показатели в сравнении с новорожденными изменяются в сторону их увеличения более интенсивно у лиц мужского пола. В ходе исследования выявлена положительная корреляционная связь сред- ней силы в группе мальчиков грудного возраста между антропометрическими данными и объемными параметрами, по скоростным показателям корреляционной связи выявлено не было. Выявлены статистически значимые различия между центральными тенденциями двух независимых выборок мальчиков и девочек грудного возраста и новорожденными по изучаемым показателям сердца, кроме фракции выброса.

\section{В.А. Васильев, К.А. Мурейси, Р.В. Басий, И.П. Вакуленко, И.В. Коктышев}

ГОО ВПО «Донецкий национальный медицинский университет имени М. Горького», Донецк

\section{МОРФОФУНКЦИОНАЛЬНЫЕ ОСОБЕННОСТИ? СТРОЕНИЯ СЕРДЦА У ДЕТЕЙ ГРУДНОГО ВОЗРАСТА ПО ДАННЫМ УЛЬТРАЗВУКОВОГО ИССЛЕДОВАНИЯ}

С каждым годом медицина принципиально меняется, и в основе этого лежит стремительное развитие аппаратной диагностики. Но, несмотря на множество работ, посвященных сердечно-сосудистой системе, и опубликованных эхокардиографических данных, они являются противоречивыми и не учитывают особенности конкретного региона. Целью данного исследования было установление количественных показателей объемных и гемодинамических параметров сердца и магистральных сосудов в области его основания у детей грудного возраста Донецкого региона. Исследования проведены на 80 детях в возрасте от одного месяца до года, у которых отсутствовали признаки заболеваний сердечно-сосудистой системы. Они были поделены на две группы по половому признаку. Первую группу составили 42 ребенка мужского пола, вторую группу - 38 детей женского пола. Методом ультразвукового исследования в М- и В-режимах измеряли и оценивали такие показатели, как конечный диастолический и конечный систолический объемы, фракцию выброса. Допплеровским методом исследовали скорость аортального, митрального, легочного и трикуспидального потоков и их градиент давления. В ходе проделанной работы были определены объемные и скоростные количественные показатели сердца мальчиков и девочек грудного возраста. Проведен корреляционный анализ исследуемых показателей, установлена корреляционная связь средней силы между антропометрическими данными и объемными параметрами сердца обеих исследуемых групп.

Ключевые слова: ультразвуковое исследование сердца, аорта, дети от одного месяца до года.

\section{V.A. Vasiliev, K.A. Mureisy, R.V. Basiy, I.P. Vakulenko, I.V. Koktyshev}

SEI HPE «M. Gorky Donetsk National Medical University», Donetsk

\section{ULTRASOUND GUIDED MORPHOFUNCTIONAL CHARACTERISTICS OF HEART STRUCTURE OF CHILDREN OF THE AGE FROM 4 WEEKS TILL 12 MONTHS}

Every year, medicine is fundamentally changing, and at the heart of this is the rapid development of hardware diagnostics. But, despite the many works devoted to the cardiovascular system and published echocardiographic data, they are contradictory and do not take into account the characteristics of a particular region. The purpose of this study was to establish quantitative indicators of volumetric and hemodynamic parameters of the heart and major vessels in the area of its base in infants of the Donetsk region. The studies were conducted on 80 children aged from one month to a year who did not have signs of diseases of the cardiovascular system. They were divided into two groups by gender. The first group consisted of 42 male children, the second group - 38 female.
By the method of ultrasound investigation in M- and Bmodes, such indicators as final diastolic and final systolic volumes, ejection fraction were measured and evaluated. The Doppler method was used to study the rate of aortic, mitral, pulmonary and tricuspid flows and their pressure gradient. In the course of the work, volume and speed quantitative indicators of the heart of boys and girls of infancy were determined. A correlation analysis of the studied indicators was carried out, a correlation relationship of the average strength between the values of both studied groups was established.

Key words: ultrasound examination of the heart, aorta, children from one month to a year. 


\section{ЛИТЕРАТУРА}

1. Владимирская М. А., Плахова В. В., Барышникова И. Ю. Трехмерная эхокардиография в оценке деталей анатомии врожденной патологии атриовентрикулярных клапанов. Бюллетень НЦССХ им. А. Н. Бакулева РАМН Сердечно-сосудистые заболевания. 2012; 13 (S3): 8.

2. Галявич А. С., Рафиков А.Ю., Сайфуллина Г.Б. Сравнительный анализ данных эхокардиографии, мультиспиральной копьютерной томографии и перфузионнной сцинтиграфии миокарда в оценке объема и фракции выброса левого желудочка. Казанский медицинский журнал. 2013; 94 (1): 39-43.

3. Воробьев А. С. Амбулаторная эхокардиография у детей : руководство для врачей Санкт-Петербург : СпецЛит.; 2010.543.

4. Грибанов А. В., Крайнова И.Н. Морфофункциональное состояние левого желудочка сердца у школьников приполярного региона. Вестник Поморского университета. $2011 ; 4: 40-46$.

5. Пшеничная, Е. В. Характеристика сердечного ритма у условно- здоровых мальчиков- подростков предпризывного возраста Донецкого региона Практическая медицина. 2017; 10 (111): 71-74.

6. Mawad W, Drolet C, Dahdah N, Dallaire F. A review and critique of the statistical methods used to generate reference values in pediatric echocardiography. J. Am. Soc. Echocardiogr. 2013; 26: 29-37.

7. Picazo-Angelin B, Zabala-Argüelles J. I, Anderson R. H., Sánchez-Quintana D. Anatomy of the normal heart: The basis for understanding echocardiography Ann. Pediatr. Cardiol. 2018; 11 (2): 164-173.

\section{REFERENCES}

1. Vladimirskaya M. A., Plakhova V. V., Bary`shnikova I. Yu. Trekhmernaya e khokardiografiya v oczenke detalej anatomii vrozhdennoj patologii atrioventrikulyarny`kh klapanov [Three-dimensional echocardiography in assessing the details of the anatomy of the congenital pathology of atrioventricular valves]. Byulleten ` $\mathrm{NCzSSKh} \mathrm{im.} \mathrm{A.}$ N. Bakuleva RAMN Serdechno-sosudisty`e zabolevaniya. 2012; 13 (S3): 8 (in Russian).

2. Galyavich A. S., Rafikov A.Yu., Sajfullina G.B. Sravnitel`ny j analiz danny`kh e`khokardiografii, mul'tispiral`noj kop yuternoj tomografii i perfuzionnnoj sczintigrafii miokarda v oczenke ob`ema i frakczii vy`brosa levogo zheludochka [Comparative analysis of echocardiography, multispiral computer tomography and perfusion myocardial scintigraphy in assessing the volume and ejection fraction of the left ventricle ]. Kazanskij mediczinskij zhurnal. 2013; 94 (1): 39-43 (in Russian).

3. Vorob`ev A. S. Ambulatornaya e`khokardiografiya u detej : rukovodstvo dlya vrachej [Ambulatory echocardiography in children: a guide for doctors]. Sankt-Peterburg : SpeczLit.; 2010. 543 (in Russian).

4. Gribanov A. V., Krajnova I.N. Morfofunkczional'noe sostoyanie levogo zheludochka serdcza u shkol 'nikov pripolyarnogo regiona [Morphofunctional state of the left ventricle of the heart in students of the circumpolar region]. Vestnik Pomorskogo universiteta. 2011; 4: 40-46 (in Russian).

5. Pshenichnaya, E. V. Kharakteristika serdechnogo ritma u uslovno- zdorovy`kh mal'chikov- podrostkov predprizy`vnogo vozrasta Doneczkogo regiona [Heart rate characteristics in conditionally healthy teenage boys of the business-like age of the Donetsk region]. Prakticheskaya mediczina. 2017; 10 (111): 71-74 (in Russian).

6. Mawad W, Drolet C, Dahdah N, Dallaire F. A review and critique of the statistical methods used to generate reference values in pediatric echocardiography. J. Am. Soc. Echocardiogr. 2013; 26: 29-37.

7. Picazo-Angelin B, Zabala-Argüelles I. I, Anderson R. H., Sánchez-Quintana D. Anatomy of the normal heart: The basis for understanding echocardiography Ann. Pediatr. Cardiol. 2018; 11 (2): 164-173. 